\title{
Patients repeatedly admitted to psychiatric wards
}

\author{
Mavis Evans, Senior Registrar; David Rice, Senior House Officer; and \\ Christina RouTh, Registrar, Directorate of Psychiatry, Wirral Hospital NHS Trust, \\ Bebington, Wirral L63 4JY
}

Many admissions to general adult psychiatric beds occur as emergencies due to social crisis or relapse of psychiatric illness. Other admissions are planned to arrest or reverse deterioration. Length of stay varies depending on reason for admission and adequacy of support in the community. Rapid readmission or failed discharge, defined as readmission within three months, has been linked with multiple previous admissions and personality factors (Jones, 1991).

All units have a few "revolving door" patients (Rollin, 1965) who are very frequently readmitted although their illnesses and social circumstances do not appear to differ markedly from others who can live successfully in the community for longer periods. We defined "revolving door" patients as those admitted six or more times within 12 months.

The aim of this study was to identify factors which would differentiate those patients admitted frequently from those remaining in the community for longer periods.

\section{The study}

A retrospective review of admissions over 12 months was carried out on two acute psychiatric wards in a District General Hospital (DGH), a 10 bed high dependency ward and a 25 bed medium dependency ward. Patients are transferred between these adjacent wards as necessary, allowing disturbed behaviour to be controlled with the minimum of sedation. The DGH serves a catchment population of approximately 360,000 , with 90 adult medium dependency beds and 20 adult high dependency beds. The catchment area is mixed rural and urban with a level of unemployment higher than the national average.

Case-note review was undertaken on the "revolving door" patients. Because it was noticed that these patients had a history of frequent discharge against medical advice (DAMA), a further review of all recorded DAMA on the wards over the same period was carried out, with case-note review of all patients.

\section{Findings}

There was a total of 440 patients admitted 573 times. Sixty-two ( 30 male, 32 female), $14 \%$ of the sample, were readmitted within three months. Three of these, all male, were readmitted over six times during the 12 month period of the study, and classified as "revolving door" patients.

Case 1: male, b. 1966. Diagnosis: schizophrenia, drug abuse; 8 admissions in study period, length of admissions 4-48 days, time between admissions 6-83 days. Usually hallucinated, sometimes threatening or violent behaviour on admission, settles quickly on the ward. Hallucinations resistant to neuroleptics. DAMA on three occasions. Poor compliance with after-care programmes and medication post discharge.

Case 2: male, b. 1947. Diagnosis: bipolar affective disorder, alcohol abuse; 8 admissions in study period, length of admissions 1-24 days, time between admissions 10-96 days. Usually hypomanic relapses. DAMA on five occasions, detained formally (Section 2) once. Established on depot but poor compliance with after-care programme.

Case 3: male, b. 1970. Diagnosis: affective disorder, alcohol and drug abuse; 7 admissions in study period, length of admissions 1-37 days, time between admissions 1-68 days. DAMA on four occasions. Very short admissions precluded proper investigations or implementation of treatment programme. Most admissions precipitated by suicidal threats. Used alcohol and drugs on the ward. Poor compliance with after-care programmes.

The majority of the readmissions in these three cases $(18 / 23)$ were self or GP referrals to a junior doctor. None were assessed by the consultant or senior registrar prior to admission. In five of the 13 recorded DAMA incidents, the patient left the ward without assessment by a medical officer.

The 32 patients who were recorded as DAMA over this period showed in some cases a pattern of behaviour with repeated episodes during their psychiatric history of leaving hospital against advice. Some would then be compliant with follow-up in the community, others would refuse contact until the next crisis, still others have as yet had no further contact. The psychiatric diagnoses that were linked with DAMA as a pattern of behaviour were alcohol abuse, personality disorder, and anorexia nervosa. Patients with schizophrenia or affective disorders were divided between those repeating DAMA and 
those in whom it was an isolated event. There was no link with age or sex, the average age of both the groups being 37 years (unique episodes) or 38 years (pattern of behaviour).

\section{Comment}

The main factors associated with the "revolving door" patients were non-compliance with treatment and frequency of DAMA. Assessment before DAMA considers whether or not the patient is detainable. DAMA usually occurs "out of hours", so assessment is likely to be carried out by the junior doctor on call. It is not the policy on many units to discuss such patients with a more senior doctor unless an element of risk is obvious and formal detention is being considered. These patients quickly become known to medical and nursing staff on the unit concerned. Once a patient becomes notorious as a "revolving door" patient, this may influence attitudes towards him of staff, both on the ward (making the chances of him discharging himself against medical advice stronger) and in the community (increasing the chances of early readmission). For good reasons the criteria for detention are strict; but if this results in allowing a patient to discharge against medical advice before he is able to survive any length of time in the community, this in itself is detrimental to the patient's health. While a treatment order can keep the patient in hospital for longer periods, it cannot be used in the community for any length of time. The consistent non-compliance shown by these patients could probably only be controlled by a community treatment order were one to exist.

All three "revolving door" patients had had a time of illness prior to this unstable period with admissions at a more "usual" rate. Indeed, two of these three patients have since had relatively lengthy periods in the community and appear to have reverted back to their previous pattern of illness. It was not possible to identify any social cause for the change in illness pattern, nor did the illness itself appear to be more severe when comparing signs and symptoms on admission. Compliance with treatment during this unstable period would be more likely to produce and maintain remission while social and psychological rehabilitation could also be carried out.

Crisis intervention teams are able to prevent some admissions by giving short term intensive support in the community to the patients or their carers. In the three cases described support in the community was not accepted.

Alcohol and/or drug abuse was present in all the three cases described, and was found in patients in whom DAMA was a pattern of behaviour. However, these are not uncommon findings in psychiatric patients. Another indication of likelihood to DAMA was if the patient had done so on a previous admission.

\section{Conclusion}

There is a small group of psychiatric patients for whom the current services available are not providing successful care. Discussion of all potential DAMA incidents with the appropriate consultant, as is recommended in the Code of Practice before Section 5(2) is implemented, may enable a treatment programme to be established in hospital. Compliance in the community is more likely to occur if the programme has been established prior to discharge.

It is important that these people who need help in times of crisis but will not accept it otherwise are not forgotten in future service developments.

\section{References}

JoNES, E. (1991) Audit in psychiatry: "failed discharges". Psychiatric Bulletin, 15, 26-27.

Roluin, H. R. (1965) Unprosecuted mentally abnormal offenders. British Medical Journal, 831-835. 